\title{
Invited Expert Opinion papers on mechanical circulatory support
}

\author{
Pavan Atluri, MD, ${ }^{a}$ and Leora B. Balsam, $\mathrm{MD}^{\mathrm{b}}$
}

In recent and upcoming issues of the Journal, our readers will have access to a series of Invited Expert Opinion papers in the field of mechanical circulatory support (MCS) with the goal of providing focused expertise within this everevolving space. Taken together, these papers paint a broad-strokes picture of the MCS landscape, focusing on areas of development, change, and innovation. Topics in both short-term MCS and long-term MCS were carefully chosen and are briefly highlighted herein.

In the field of short-term MCS, venoarterial extracorporeal membrane oxygenation (VA-ECMO) in adults has gained momentum in the treatment of cardiogenic shock (CS). The group from Minnesota ${ }^{1}$ describes their institutional approach for the management of CS, which favors early implementation of VA-ECMO. Once on VA-ECMO, the obvious goal is to restore adequate perfusion and maintain end-organ function while minimizing complications of the therapy. The authors discuss their strategy for transitioning off of ECMO, including when to explant, bridge to other advanced therapies, or consider withdrawal of support.

Standard criteria for the use of VA-ECMO for CS are lacking for many reasons. Just as the Minnesota group describes their triggers for initiating VA-ECMO, Rao and Billia $^{2}$ from the Toronto group describe their program's approach within the Canadian Nationalized Health Care System. It is important to acknowledge that VA-ECMO for CS is not yet a universal "standard of care," as many institutions remain with limited or no access to it, given human resources and other resource limitations. But for those who do, the decision of how to use this resource can be quite challenging. For those entrenched in the field of transplantation, the concept of restricting access to a scarce resource is not new, but still the burden of this decisionmaking can be heavy for clinicians, patients, and their families. Given the lack of general guidelines and consensus, difficulty remains in ECMO resource allocation and withdrawal.

From the a Division of Cardiovascular Surgery, Department of Surgery, University of Pennsylvania, Philadelphia, Pa; and ${ }^{\mathrm{b}}$ Division of Cardiac Surgery, UMass Memorial Medical Center, Worcester, Mass.

Received for publication March 19, 2021; revisions received March 19, 2021; accepted for publication March 23, 2021; available ahead of print April 20, 2021

Address for reprints: Leora B. Balsam, MD, Division of Cardiac Surgery UMass Memorial Medical Center, University Campus, 55 Lake Ave, North Worcester, MA 01655 (E-mail: leora.balsam@umassmemorial.org).

J Thorac Cardiovasc Surg 2021;162:140-2

$0022-5223 / \$ 36.00$

Copyright (c) 2021 by The American Association for Thoracic Surgery

https://doi.org/10.1016/j.jtcvs.2021.03.107

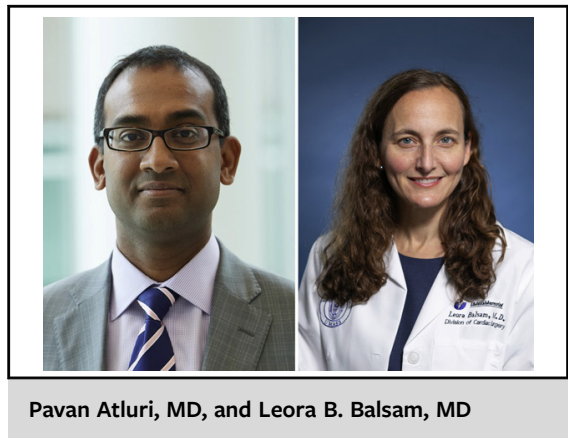

CENTRAL MESSAGE

Major developments in the field of mechanical circulatory support are highlighted in a series of Expert Opinion papers in the Journal.

The Maryland group describes another facet of ECMO therapy in their paper, the use of hybrid and parallel ECMO circuits. ${ }^{3}$ The terminology "hybrid" refers to a combination of VA- and venovenous ECMO, typically implemented when the initial strategy of VA-ECMO or venovenous ECMO provides inadequate support. Importantly, registry data from Extracorporeal Life Support Organization inform us that these configurations are rarely used, and when they are required, outcomes are worse than with nonhybrid ECMO configurations. The practice of using parallel ECMO circuits is anecdotal, and given the resource consumption and complexity of conventional ECMO therapy, the idea of using 2 circuits on 1 patient may not gain significant traction.

An interesting topic in short-term MCS is how to achieve left ventricular (LV) unloading, a particular challenge in patients on VA-ECMO. The importance of LV unloading for the reduction of wall stress and myocardial oxygen demand has been established in experimental models of acute myocardial infarction (AMI) as a means of limiting infarct propagation and adverse ventricular remodeling. Experimental animal models have shown a clear reduction in infarct size with LV unloading after myocardial infarction. This experimental finding forms the basis for a new strategy of early LV unloading in the treatment of AMI, measured as the "door-to-unloading" time. Goodwin and Selzman ${ }^{4}$ from Utah describe the science behind new clinical trials that 
compare a strategy of initial LV unloading with a percutaneous ventricular assist device (VAD) followed by percutaneous coronary intervention to immediate percutaneous coronary intervention for AMI.

The Minnesota group summarizes the current state-ofthe-art in durable long-term VADs in another Invited Expert Opinion. ${ }^{5}$ The field has moved away from axial flow devices to miniaturized centrifugal pumps, with improvement in some of the complications of earlier-generation devices. Nevertheless, the adverse event profile of the therapy still limits broader application to patients with earlier stages of heart failure. There has been movement away from the labels "bridge to transplantation" or "destination therapy," both in Food and Drug Administration clinical trial design and national coverage decisions from the Centers for Medicare and Medicaid Services.

In another Expert Opinion statement paper, Estep, Soltesz, and Cogswell ${ }^{6}$ discuss their early observations with the new heart allocation system. Following a careful analysis of the current research on the new allocation system that took effect in October 2018, they note both improved waitlist mortality and preservation of mid-term (6-month) survival. Yet, there has been an increase in the use of ECMO, intra-aortic balloon pumps, and temporary MCS, which has shifted transplants to a "more ill" cohort. In addition, there has been a parallel increase in allograft ischemic time, presumably related to greater organ sharing with the need for increased travel times. Those patients with VADs are disproportionately becoming a smaller fraction of the general transplant pool, likely due to a disadvantage in priority relative to the other subgroups listed for transplant. The decreased use of VADs as a "bridge to transplant," as previously discussed, may further be contributing to this decline in patients with VADs going on to transplant. As greater time elapses from institution of the allocation change, its long-term impact will become clearer.

It has been by some degree of science, trial, and error that we have learned how to use our existing durable VADs most effectively. In their Expert Opinion paper, Smood and colleagues ${ }^{7}$ from Penn discuss their approach to managing aortic regurgitation and mitral regurgitation (MR) in patients undergoing durable left ventricular assist device (LVAD) implantation. For aortic regurgitation, they recommend valve replacement with a bioprosthesis for grades more than mild. The alternative of valve repair with the Park's stitch has been used widely but may have disadvantages of worse durability. The population of patients who may benefit from mitral repair for severe MR during LVAD implantation is less clearly established, since most often MR improves significantly with LV unloading. For patients with anticipated ventricular recovery, the authors emphasize the importance of concomitant mitral repair. There also appears to be a subset of patients who continue to have severe
MR, pulmonary hypertension, and right ventricular failure after durable LVAD implantation, but we currently lack reliable means to identify these patients preoperatively.

Perhaps the ultimate goal of VAD therapy is the total recovery of myocardial function following a period of unloading. While this pursuit has been extensively studied and investigated, optimal strategies and outcomes remain elusive. In their Expert Opinion paper, Faerber and Doenst ${ }^{8}$ elegantly discuss the potential biologic and mechanistic pathways involved in myocardial recovery. Furthermore, they highlight the subset of patients who currently should be considered for recovery as well as the strategies for their support. Most importantly, they discuss the essential elements of goaldirected medical therapy and serial evaluation of myocardial function based on clearly defined weaning protocols. For those patients suitable for VAD explant, techniques for device explant and deactivation are clearly laid out.

While we have provided a very broad overview of these excellent Expert Opinion papers, careful review of the content will provide valuable insight to our readers, along with opportunity to apply these concepts in their own clinical practices.

\section{Conflict of Interest Statement}

The authors reported no conflicts of interest.

The Journal policy requires editors and reviewers to disclose conflicts of interest and to decline handling or reviewing manuscripts for which they may have a conflict of interest. The editors and reviewers of this article have no conflicts of interest.

\section{References}

1. Qi SS, Shaffer AW, Cogswell R, John R. Short-term mechanical circulatory support: transitioning the patient to the next stage. J Thorac Cardiovasc Surg Open. 2020;2:29-34.

2. Rao V, Billia F. When NOT to use short-term mechanical circulatory support. $J$ Thorac Cardiovasc Surg Open. 2020;3:106-10.

3. Shah A, Dave S, Goerlich CE, Kaczorowski DJ. Hybrid and parallel extracorporeal oxygenation circuits. J Thorac Cardiovasc Surg Tech. February 24, 2021 [Epub ahead of print].

4. Goodwin ML, Selzman CH. Take a load off: unloading and reperfusion. J Thorac Cardiovasc Surg. July 25, 2020 [Epub ahead of print].

5. Shaffer A, Cogswell R, John R. Future developments in left ventricular assist device therapy. J Thorac Cardiovasc Surg. September 16, 2020 [Epub ahead of print].

6. Estep J, Soltesz E, Cogswell R. The new heart transplant allocation system: early observations and urgent heart transplant and mechanical circulatory device considerations. J Thorac Cardiovasc Surg. 2021;161:1839-46.

7. Smood B, Han JJ, Helmers MR, Atluri P. Mitral and aortic valve surgery during LVAD implantation. J Thorac Cardiovasc Surg. March 18, 2021 [Epub ahead of print].

8. Faerber G, Doenst T. Ventricular assist device-promoted recovery and technical aspects of explant. J Thorac Cardiovasc Surg Tech. February 23, 2021 [Epub ahead of print].

Key Words: mechanical circulatory support, ECMO, extracorporeal membrane oxygenation, ventricular assist device, organ allocation, heart 


\section{Mechanical Circulatory Support: Recent Articles from AATS Journals}

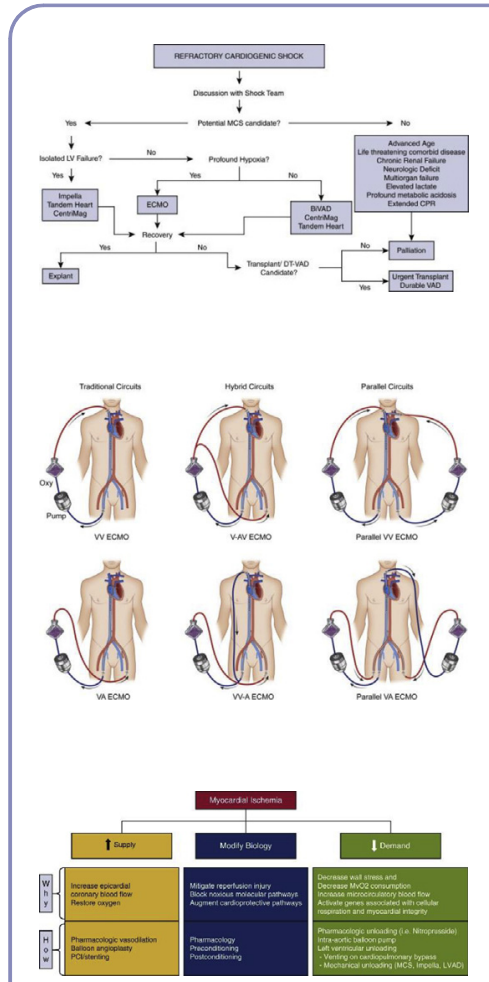

JTCVS OPEN: When NOT to use short term mechanical circulatory support. Rao V, Billia F. J Thorac Cardiovasc Surg Open. 2020;3:106-110.

Commentary: It's tempting to jump down the rabbit hole. Kilic A. J Thorac Cardiovasc Surg Open. 2020;3:111.

Commentary: Futility in the age of modern mechanical circulatory support. Schutz A, Ghanta R. J Thorac Cardiovasc Surg Open. 2020;3:112-113.

JTCVS TECHNIQUES: Hybrid and parallel extracorporeal membrane oxygenation circuits. Shah A, Dave S, Goerlich CE, Kaczorowski DJ. J Thorac Cardiovasc Surg Tech. 2021 [In press].

Commentary: More is better: Hybrid and parallel extracorporeal membrane oxygenation circuits. Pagani F. J Thorac Cardiovasc Surg Tech. 2021 [In press].

Commentary: Never let semantics get in the way of a good idea. Guo MH, Rubens FD. J Thorac Cardiovasc Surg Tech. 2021 [In press].

JTcvs: Take a load off: Unloading and reperfusion. Goodwin ML, Selzman CH. J Thorac Cardiovasc Surg. 2021 [In press].

Commentary: Long journey and a heavy load. Timek T. J Thorac Cardiovasc Surg. 2021 [In press].

Commentary: "The weight" will be worth it: Avoiding premature judgment on the success or failure of left ventricular unloading in mitigating reperfusion injury. Brener MI, Burkhoff D. J Thorac Cardiovasc Surg. 2021 [In press].

Commentary: What is behind the door to unloading? Glower DD. J Thorac Cardiovasc Surg. 2021 [In press].

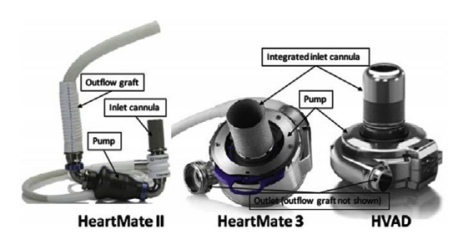

JTCVS: Future developments in left ventricular assist device therapy. Shaffer A, Cogswell R, John R. J Thorac Cardiovasc Surg. 2021 [In press].

Commentary: Better pumps, better patients, better physicians? Future developments in left ventricular assist device therapy. Silvestry SC. J Thorac Cardiovasc Surg. 2021 [In press].

Commentary: Managing the native heart in patients supported with durable left ventricular assist devices. Milano CA. J Thorac Cardiovasc Surg. 2021 [In press].

Commentary: Left ventricular assist device therapy: The time is now. Kilic A. J Thorac Cardiovasc Surg. 2021 [In press]. 